\title{
In Situ Observation of Ag Nanoparticle Catalyzed Oxidation of Carbon Nanotubes in an Aberration-corrected Environmental TEM
}

\author{
Yonghai Yue ${ }^{1,2}$, Datong Yuchi ${ }^{3}, \mathrm{Jia} \mathrm{Xu}^{3}, \mathrm{Lin} \mathrm{Guo}^{2}$ and Jingyue Liu ${ }^{1}$ \\ 1. Department of Physics, Arizona State University, Tempe, Arizona 85287, USA \\ 2. School of Chemistry and Environment, Beihang University, Beijing 100191, PR China \\ 3. School for Engineering of Matter, Transport and Energy, Arizona State University, Tempe, Arizona \\ 85287, USA
}

Carbon nanotubes (CNTs) [1], with their unique physicochemical properties, have attracted broad attention due to their potential applications [2]. A recent in situ study on the oxidation of different types of CNTs has revealed that the initiation temperature of CNT oxidation depends on the wall thickness of the CNTs [3]. Single- and double-wall CNTs can oxidize at lower temperatures and multi-wall CNTs can be stable in oxygen even at temperatures as high as $520{ }^{\circ} \mathrm{C}$ [3]. With the use of catalyst nanoparticles to facilitate the oxidation processes the initiation temperature and the degree of carbon oxidation can be drastically changed [4]. Understanding the behavior of catalyzed oxidation processes is critical to developing better catalysts for combustion of particulates including oxidation of soot particles [5]. We report here the in situ investigation of Ag catalyzed oxidation of multi-wall CNTs inside an aberration-corrected environmental TEM (AC-ETEM) with the goal of probing the nature of the catalyzed oxidation processes and thus developing better understanding of combustion catalysts.

The CNTs, which were prepared via a CVD method, were suspended in ethyl alcohol and a drop of the solution was then transferred to a holey-carbon coated TEM grid. The FEI Titan G2 AC-ETEM, operating at $80 \mathrm{KV}$, was used to carry out the in situ oxidation experiment. The electron dose rate was kept low enough so that the electron beam exerts minimum influence on the integrity of the experimental results. A Gatan Inconel heating holder was used and the images were recorded by a standard CCD camera. To clean the CNTs, the TEM grid was heated to $450^{\circ} \mathrm{C}$ in $2 \mathrm{mbar}_{2}$ for 45 min inside the ETEM. Repeated experiments confirmed that such treatment did not oxidize the multi-wall CNTs. The CNTs were then loaded with $\mathrm{AgNO}_{3}$ aqueous solution and was then reduced inside the ETEM in $2 \mathrm{mbar}_{2}$ at $250^{\circ} \mathrm{C}$ for 2 hours, resulting in the formation of crystalline $\mathrm{Ag}$ nanoparticles. The electron beam was blanked during the sample treatment processes.

Figure 1 shows AC-ETEM images of the CNTs after the sample treatment processes. Ag nanoparticles, with an average diameter of approximately $5 \mathrm{~nm}$, decorated on the surfaces of the CNTs. After this initial quick examination the electron beam was blanked and the sample was oxidized in 2 mbar $\mathrm{O}_{2}$ at $250^{\circ} \mathrm{C}$ for 25 minutes. To avoid any potential ionization effects, the oxygen inside the ETEM chamber was completely purged after the oxidation reaction and before the electron beam was un-blanked [3]. The same sample region, before and after the oxidation reaction, was carefully examined and images were recorded. Fig. $1 \mathrm{~b}$ shows the same area as that of Fig. 1a but after the oxidation reaction. The CNTs were clearly oxidized wherever they were in contact with the Ag nanoparticles. Figure 2 shows another pair of images of a single CNT before and after the oxidation reaction. Based on analysis of these and many other similar images we concluded that the Ag nanoparticles clearly catalyzed the oxidation of multi-wall CNTs at temperatures as low as $250^{\circ} \mathrm{C}$, in contrast to the high temperature oxidation of CNTs without Ag nanoparticles [3]. Furthermore, the oxidation did not proceed along the length of, but across, the CNTs, a different process than that reported for the non-catalyzed oxidation of CNTs [3]. AC-ETEM 
proves to be a powerful tool for studying the oxidation processes of carbon particulates including CNTs and soot particles [6].

\section{References:}

[1] S. Iijima, Nature 354 (1991) p.56.

[2] M. S. Dresselhaus in "Carbon Nanotubes: Synthesis, Structure, Properties, and Applications", ed. M. S. Dresselhaus, (Springer, New York) p.1.

[3] A. L. Koh et al. ACS Nano 7 (2013), p.2566.

[4] A. R. Leino et al. Carbon 57 (2013), p.99.

[5] B. R. Stanmore and J. F. B. P Gilot, Carbon 39 (2001), p.2247.

[6] This work was supported by the start-up fund of the College of Liberal Arts and Sciences of Arizona State University. The authors acknowledge the use of facilities in the John M. Cowley Center for High Resolution Electron Microscopy at Arizona State University. YY acknowledges China Scholarship Council (201406025060), National Natural Science Foundation of China (51301011) and Research Fund for the Doctoral Program of Higher Education of China (20131102120053).
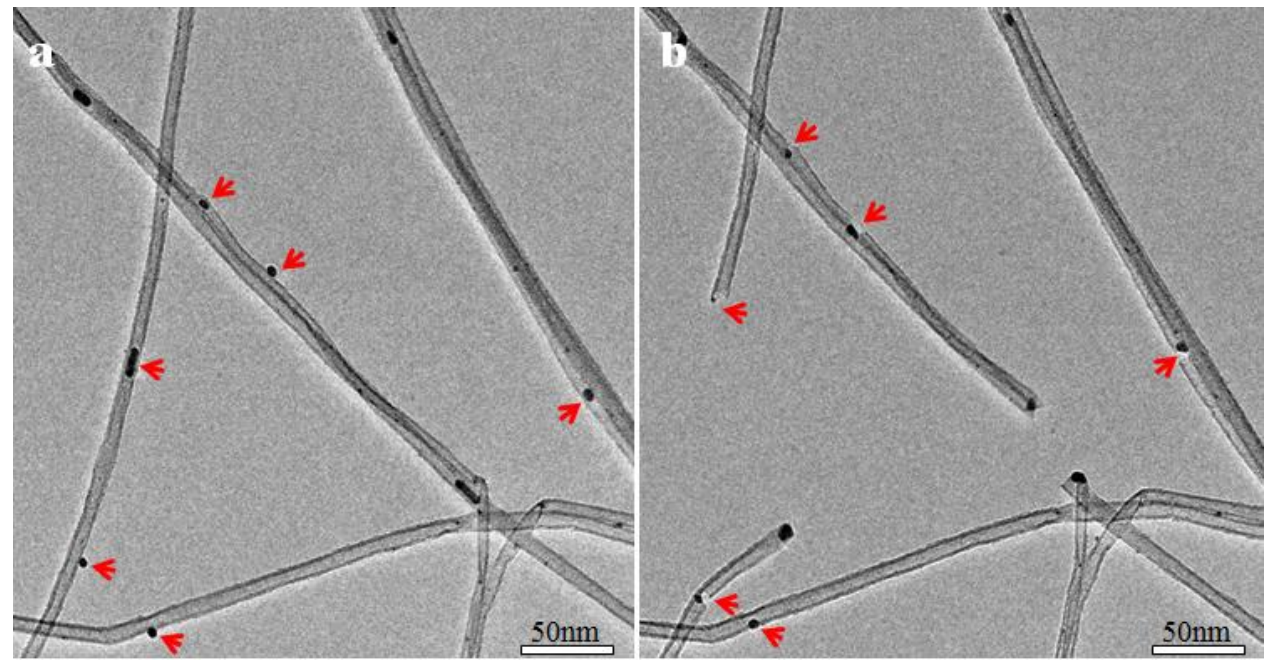

Figure 1. ETEM Images of multi-wall CNTs before (a) and after (b) oxidation at $250^{\circ} \mathrm{C}$ for 25 minutes, clearly demonstrating the Ag nanoparticle catalyzed oxidation of the multi-wall CNTs.

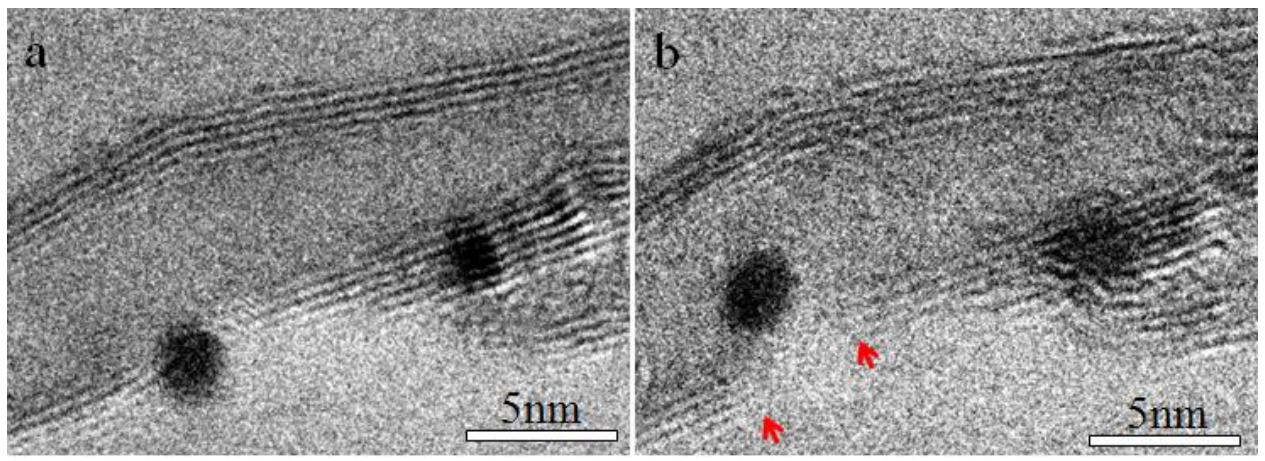

Figure 2. ETEM images of a multi-wall CNT before (a) and after (b) oxidation at $250^{\circ} \mathrm{C}$ for 25 minutes, clearly revealing that the carbon oxidation proceeded across the CNT, not along its length. 